This is an Open Access article distributed under the terms of the Creative Commons Attribution-Noncommercial License, which permits unrestricted use, distribution, and reproduction in any noncommercial medium, provided the original work is properly cited

\title{
SPICA CORONAGRAPH INSTRUMENT (SCI) FOR THE DIRECT IMAGING AND SPECTROSCOPY OF EXO-PLANETS
}

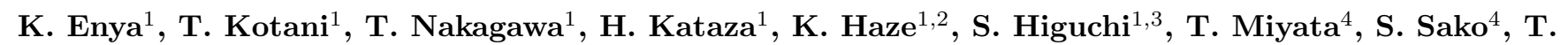
Nakamura $^{4}$, T. Yamashita ${ }^{5}$, N. Narita ${ }^{5}$, M. Tamura ${ }^{5}$, J. Nishikawa ${ }^{5}$ H. Hayano , S. Oya $^{5}$, Y. Itoh $^{6}$, M. Fukagawa ${ }^{7}$, H. Shibai ${ }^{7}$, M. Honda ${ }^{8}$, N. Baba ${ }^{9}$, N. Murakami ${ }^{9}$, M. Takami ${ }^{10}$, T. Matsuo ${ }^{11}$, S. Ida ${ }^{12}$, L. Abe ${ }^{13}$, O. Guyon ${ }^{14}$, M. Venet ${ }^{15}$, T. Yamamuro ${ }^{16}$, P. Bierden ${ }^{16}$, and SPICA coronagraph team

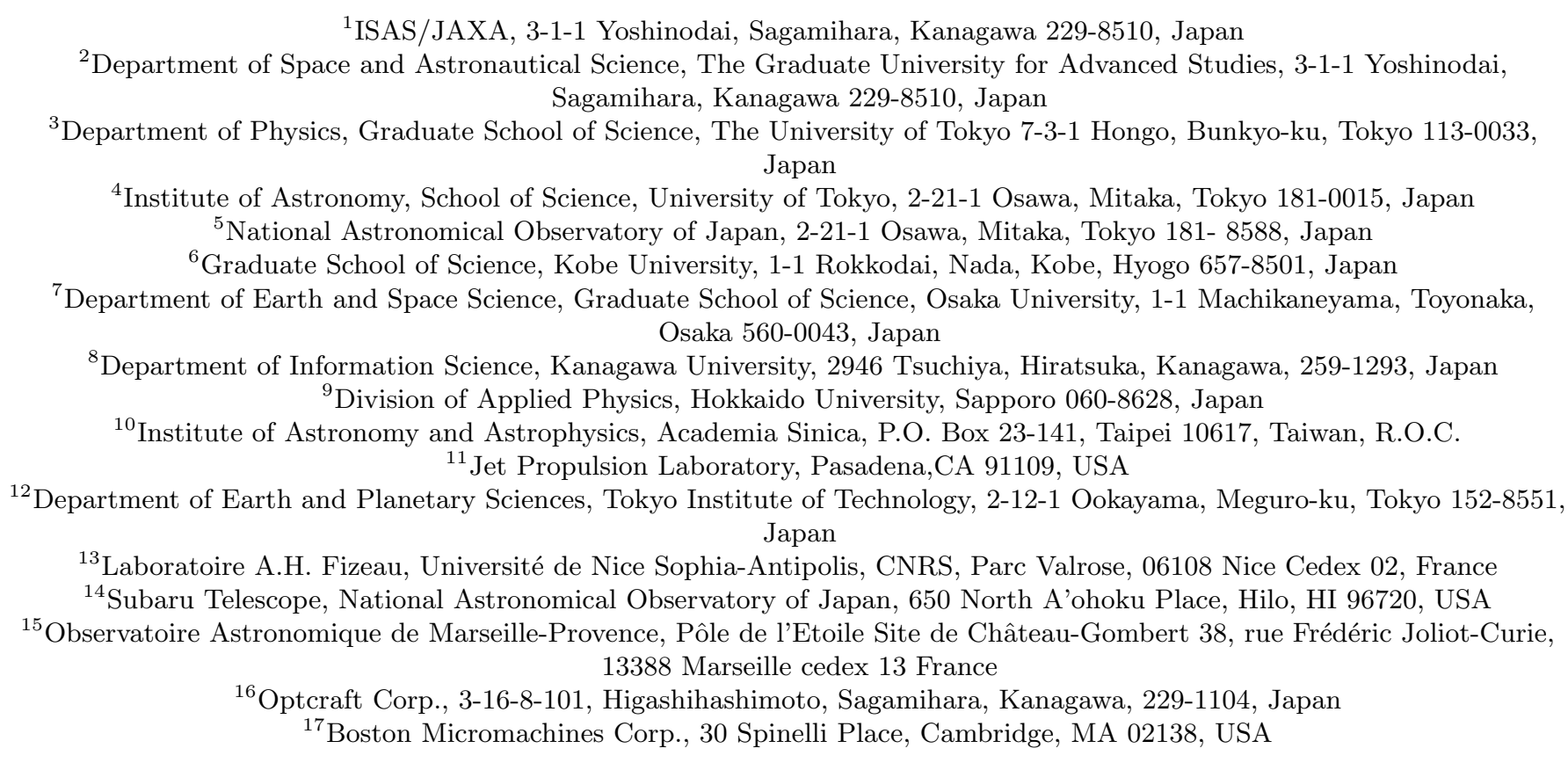

\section{ABstract}

We present the SPICA Coronagraph Instrument (SCI) for the direct imaging and spectroscopy of exo-planets.

The SPICA mission gives us a unique opportunity for high-contrast observations because of the large telescope aperture, the simple pupil shape, and the capability for infrared observations from space. The primary target of SCI is Jovian exo-planets. Using the spectroscopy mode of SCI, we will try the detection and the characterization of mid-infrared line features of the atmosphere of exoplanets. The specifications, performance and the design of the instrument are shown. The main wavelengths and the contrast required for the observations are $3.5-27 \mu \mathrm{m}$, and $10^{-6}$, respectively. We also show the progress of the development of key technology to realize SCI. Laboratory demonstration of the principle of coronagraph, realistic design and fabrication of masks, and the development of cryogenic active optics have been carried out, or are successfully ongoing. We are preparing a cryogenic chamber for the tests of the whole infrared coronagraph. A potentially important by-product of the instrument, transit monitoring for characterization of exo-planets, is also described. We expect that SCI will provide drastic progress in the understanding of various planetary systems and will be a unique capability in the SPICA era.
Key words: SPICA, coronagraph, instrument, exo-planet, transit, spectroscopy

\section{INTRODUCTION}

We consider that systematic characterization of exo-planets one of the most important issues for space science in the near future. Since the first report by Mayor \& Queloz (1995), more than 300 extra-solar planets (hereafter exoplanets) have been discovered by the radial velocity method. However, such indirect detection, measuring the influence of a planet to the parent star, usually does not provide atmospheric spectral features of the planet.

Using monitor observation of planetary transits, recently atmospheric spectral features of some exo-planets were observed. Tinetti et al. (2007), Swain, Vasisht \& Tinetti (2008) reported detection of $\mathrm{H}_{2} \mathrm{O}$ and $\mathrm{CH}_{4}$ features in exo-planet HD189733b observed by Spitzer and HST. Though this method is quite interesting, it should be noted that the targets are biased to planets very close to the parent star, "hot Jupiter". The direct observation of exo-planets spatially resolved from the parent star is the clear way to approach the characterization of exo-planets. Recently, the first direct detection of an exo-planet was finally reported by Marois et al. (2008) and Kalas et al. 


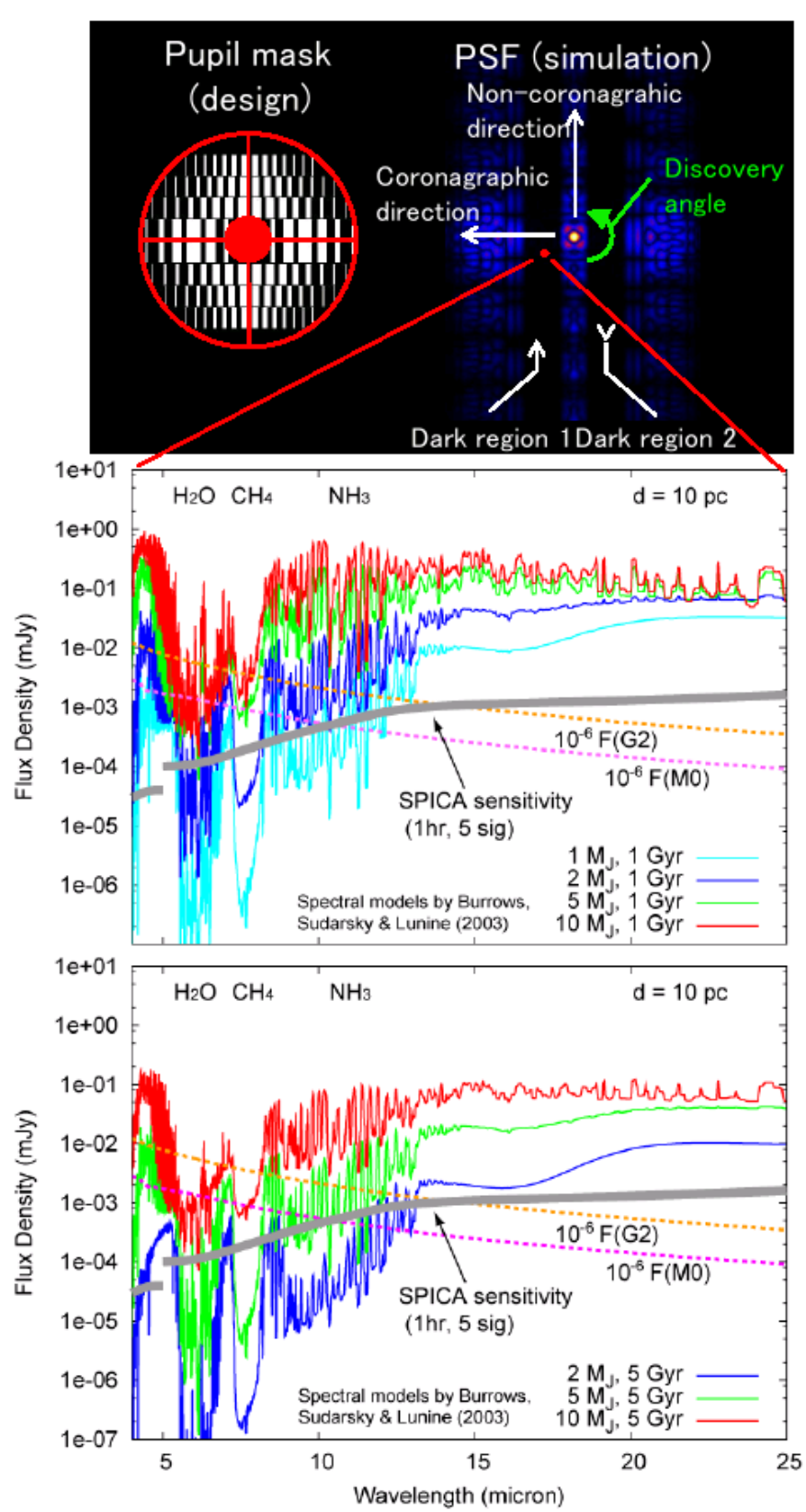

Figure 1. Top-left: An example of design of multi-barcode pupil mask for $1 D$ coronagraph. Transmissivity of white and black part of the mask is 1 and 0, respectively. Top-right: Expected PSF. Middle and bottom panel show the detection limit of SPICA and the spectrum of Jovian exo-planets with various masses and ages derived by Burrows, Sudarsky, \& Hubeny (2004). Suppressed light of the parent star is also shown by curves.

(2008). However, the observation is limited to detection and spectroscopy is not achieved.

Considering this situation, we are developing the SPICA coronagraph instrument (SCI) in order to carry out the systematic characterization of exo-planets by direct imaging and spectroscopy.

Table 1. Specifications of SCI

\begin{tabular}{|c|c|}
\hline Wavelength & $\begin{array}{l}3.5-27 \mu m \\
\text { (In } 1-3.5 \mu m, \text { SCI has sensitivity } \\
\text { though high contrast is not guaranteed). }\end{array}$ \\
\hline Observation mode & $\begin{array}{l}\text { Coronagrahpic/Non-coronagraphic, } \\
\text { Imaging/Spectroscopy }\end{array}$ \\
\hline Coronagraph & Binary-shaped pupil mask \\
\hline Contrast & $10^{-6}$ \\
\hline Inner working angle & $3.3 \lambda / D$ \\
\hline Outer working angle & $16 \lambda / D$ \\
\hline Detector & $\begin{array}{l}\text { Si:As } 1 \mathrm{k} \times 1 \mathrm{k} \text { (long wavelength channel) } \\
\text { InSb } 1 \mathrm{k} \times 1 \mathrm{k} \text { (short wavelength channel) }\end{array}$ \\
\hline Field of View & $1.0 \times 1.0 \operatorname{arcmin}^{2}$ \\
\hline Spectral resolution & $\mathrm{R}=20,200$ \\
\hline
\end{tabular}

\section{SCI AND ITS PERFORMANCE}

The specifications of SCI is shown in Table 1. SCI has the capability to provide high contrast $\left(10^{-6}\right)$ point spread function (PSF) and continuous wavelength coverage of spectroscopy, while a non-coronagraphic mode is also prepared to use in SCI thanks to the mask changer. The core wavelength region is $3.5-27 \mu \mathrm{m}$, and equipped wavelength resolution is $\sim 20$ and $\sim 200$. Figure 1 shows expected spectra of exo-planets with various masses and ages (Burrows, Sudarsky, \& Hubeny, 2004), derived by simulation with observation performance (Fukagawa, Itoh, \& Enya, 2009). SCI suppresses the parent starlight, and its spectroscopy mode reveals essentially important spectral features in the mid-infrared, $\mathrm{CH}_{4}, \mathrm{H}_{2} \mathrm{O}, \mathrm{CO}_{2}, \mathrm{CO}, \mathrm{NH}_{3}$. Jovian exoplanets around 1$)$ nearby stars $(<10 \mathrm{pc})$ and 2$)$ young and modestly old stars $(<1 \mathrm{Gyr}$ old $)$ are suitable targets of SCI. The former is suitable for detailed spatially resolved observations, and the later is suitable to understand the history of the planetary system formation. If the planets are older (e.g., sim5 Gyr old), the minimum mass of detectable planets increases. SCI is expected to make an atlas of various spectra of exo-planets as a result of the observations of $\sim 100$ s targets.

Coronagraphic observation by SCI covers "outer planets", e.g., 10 AU or farther planets from the parent star. So coronagraphic observation and observation of the planetary transit is complementary. We are planning to use SCI for the characterization of planets by transit method with non-coronagraphic mode (Yamashita, Narita, \& Enya (2009)). The advantages of SCI as a transit monitor instrument are the capability of simultaneous observations with two detectors (InSb and Si:As for thr short and long 
wavelength channels, respectively), the best pointing stability in the SPICA instruments ( 0.03 arcsec realized by internal tip-tilt mirror), and the use of a deformable mirror for defocusing to avoid saturation. Using spectrum data sets of various exo-planets obtained with SCI, we expect that our understanding for the whole planetary system will be improved drastically (please see also Abe et al., 2009). Finally we should not deny the possibility to try to detect the more challenging bio-marker, $\mathrm{O}_{3}$ with SCI.

The SPICA mission gives us a unique opportunity for high-contrast observations because of the large telescope aperture, the simple pupil shape, and the capability for infrared observations from space (Nakagawa, 2009). It should be noted that only our SCI has the potential to provide the spectral atlas of various exo-planets in the mid-infrared, because ground based telescopes don't have enough sensitivity in the mid-infrared, and JWST does not have the capability of spectroscopy on coronagraphic image. Therefore, we expect that spectroscopy with SCI will be "critical science" still in 2018. An overview of SCI is shown in Figure 2 .

\section{Development of Key technologies}

This section introduces the progress of the development of key technology to realize SCI. More detail is shown in Enya et al. (2009) and its references.

\subsection{LABORATORY DEMONSTRATION OF CORONAGRAPH}

The SPICA coronagraph has to work in the mid-infrared wavelength region in a cryogenic environment. The coronagraph should be robust against telescope pointing errors caused by vibration of the mechanical cryo-cooler system and the attitude control system of the satellite. Achromatism was also assumed to be a beneficial property to realize the spectroscopy mode. After consideration for these points, we selected a coronagraph implemented by a binary-shaped pupil mask as the primary candidate to be studied because of its physical properties and its feasibility.

The purpose of the first step of our coronagraphic experiment is the demonstration of the coronagrahpic principle. So we started experiments in air using substratebased binary-shaped pupil masks and visible laser as a light source (Figure 3). Electron beam drawing method was used to manufacture pupil masks on glass substrates. Considering convenience of fabrication, we selected a checkerboard type design of pupil mask presented by Vanderbei, Kasdin, \& Spergel (2004). Contrast of $6.7 \times 10^{-8}$ was achieved without adaptive optics. This value satisfies the requirement of SCI. It should be noted that a software LOQO presented by Vanderbei (1999) was used for optimization of shaped pupil masks.

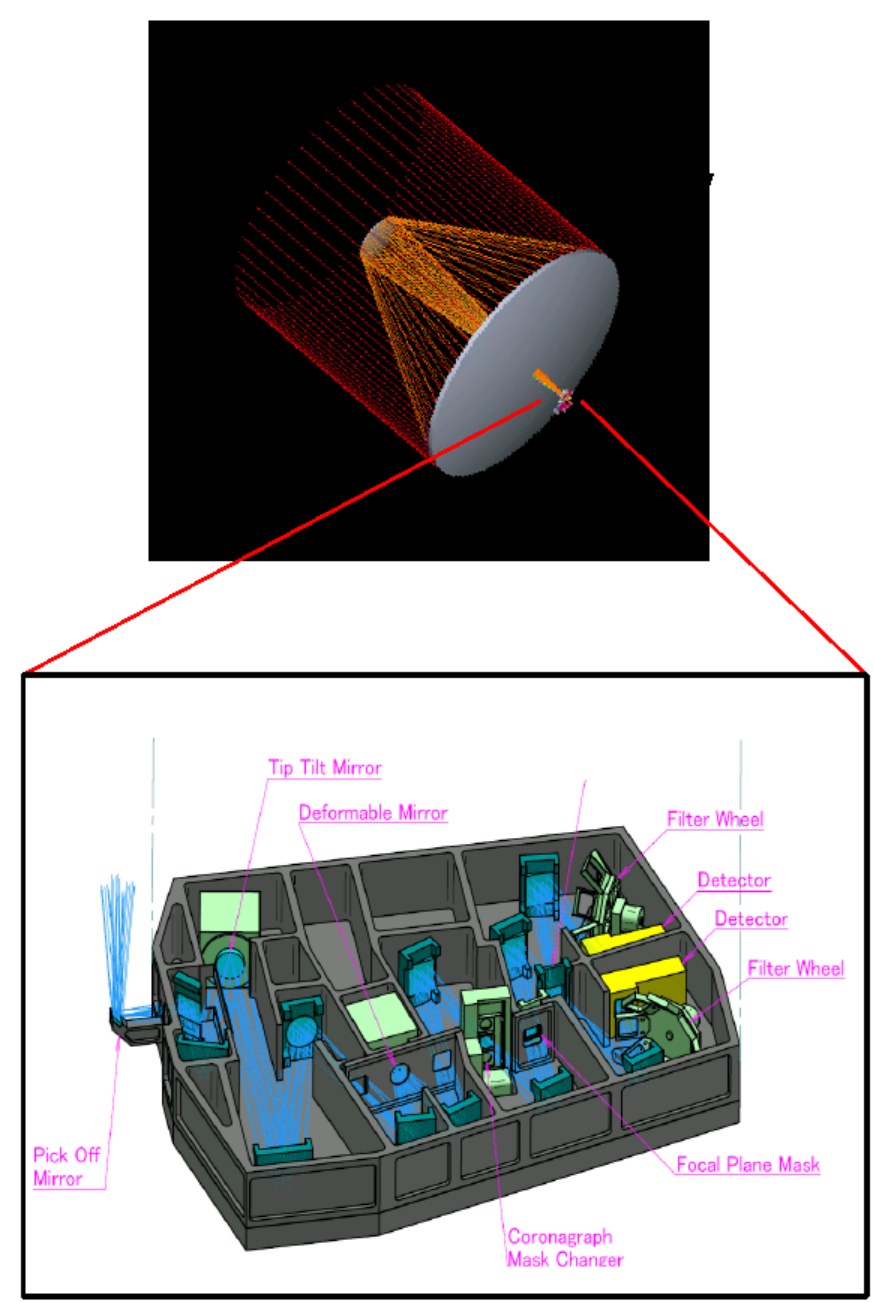

Figure 2. Overview of the design of SCI at the focal plane of the telescope.

\subsection{FREE STANDING MASK}

To realize a MIR coronagraph, shaped pupil masks with glass substrate are not useful because the substrate is not transparent in MIR wavelengths. Therefore we developed free standing masks (i.e., masks without substrate). Figure 4 shows a proto-type of a free standing (i.e., without substrate) mask of $\sim 10 \mathrm{~mm}$ size made of copper.

Quick tests of coronagraphic performance with these masks were performed using visible light. High contrast, $7 \times 10^{-7}$, was achieved as the average contrast in dark regions without adaptive optics, while contrast just close to the inner working angle (IWA) is not so high. Preparation of more extensive tests using this mask in a MIR testbed is ongoing. 

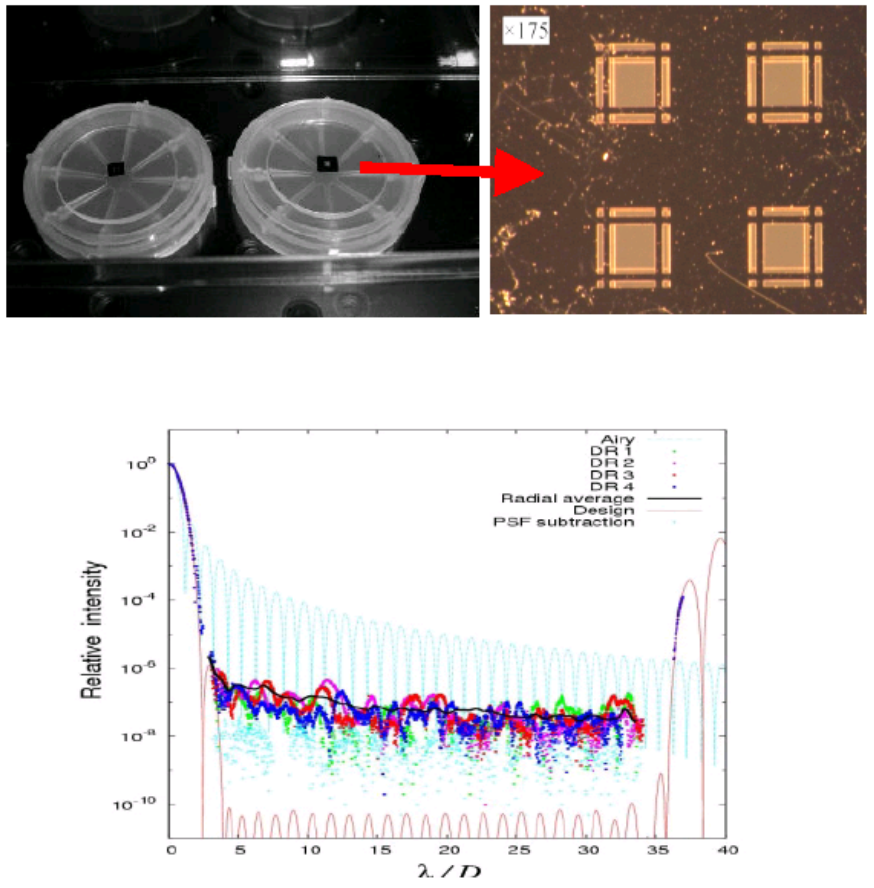

Figure 3. Top: Binary shaped pupil masks for demonstration of the principle of coronagraph method. Some checkerboard mask with $\sim 2 \mathrm{~mm}$ size were manufactured on glass substrate using electron beam drawing. Bottom: Profiles obtained as the demonstration using visible laser in a laboratory experiment. Average contrast of $6.7 \times 10^{-8}$ was achieved without adaptive optics.

\subsection{MASK FOR THE PUPIL OF SPICA}

The pupil of SPICA is obscured by the secondary mirror and its support structure of the telescope (Figure 2). So a special new design of shaped pupil mask was needed to adapt to the pupil of SPICA.

An example of our solution is shown in Figure 2. This design consists of multi-barcode masks and has coronagraphic power only in one dimension (horizontal direction in Figure 2). As a result, the specification on IWA is satisfied and a large opening angle is realized. It should be noted that the principle of a barcode mask was presented by Kasdin et al. (2005).

\subsection{Cryogenic ACtive optics}

The specification of the wave-front quality is $350 \mathrm{~nm} \mathrm{rms}$ for the SPICA telescope, while the requirement of the SCI is higher. Therefore, SCI needs an active wave-front correction which works at the temperature of work-surface of SCI (i.e., $\sim 5 \mathrm{~K}$ ).

We developed a proto-type of the cryogenic deformable mirror as shown in Figure 5. The proto-type device with 32 channels consists of Micro Electro Mechanical Systems (MEMS) of Boston Micromachines Corporation (BMC) and special substrates designed to minimize thermal stress
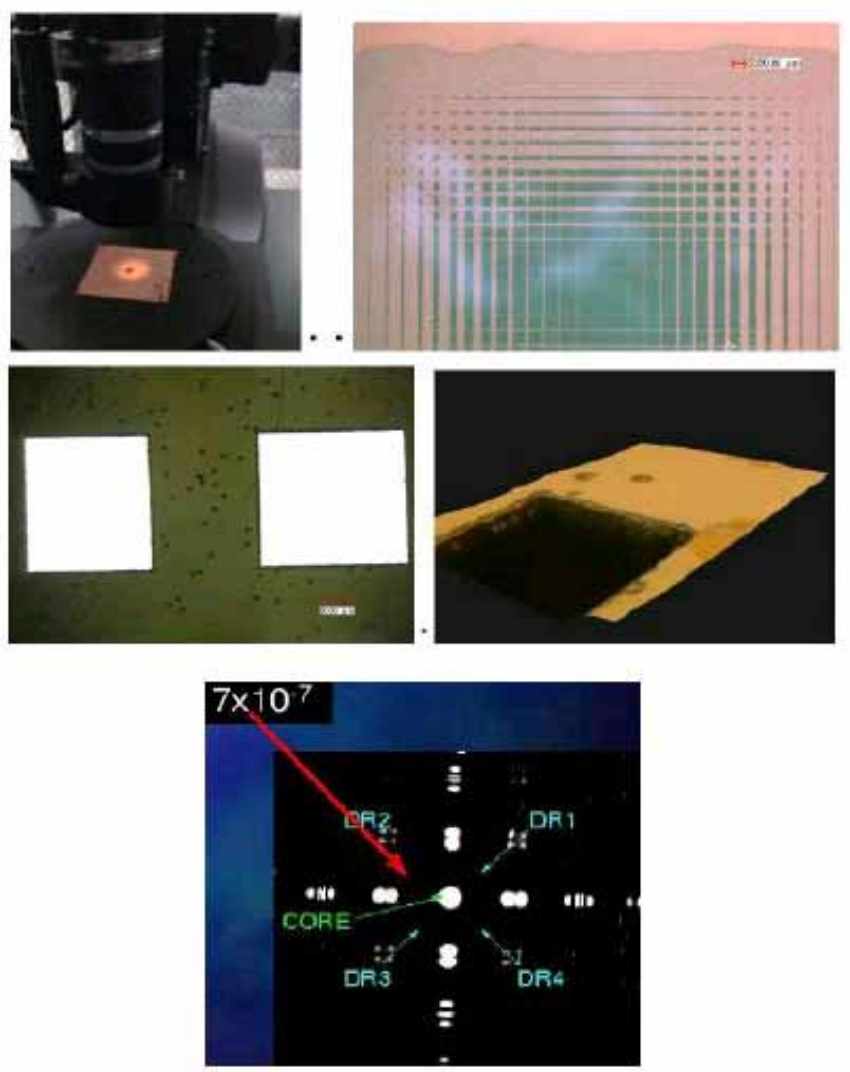

Figure 4. Top and middle: proto-type of free standing (i.e., without substrate) mask with $\sim 10 \mathrm{~mm}$ size made of copper. Bottom: Coronagraphic PSF obtained using a free standing mask and visible laser in a laboratory experiment. Average contrast of $7 \times 10^{-7}$ was achieved without adaptive optics.

caused by cooling. Figure 5 also shows the result of the demonstration of the proto-type at cryogenic environment $(95 \mathrm{~K})$. The development of a larger format deformable mirror ( 1000 channels $)$ is ongoing.

\subsection{WAVE-FRONT CORRECTION ALGORITHM}

It is an important issue how to operate a deformable mirror to correct the wave-front error. In the context of the study of such algorithm, we started experiments with visible laser and a commercially available deformable mirror of BMC. Figure 6 shows an example of the result of our wave-front correction, in which a checkerboard type pupil mask coronagraph was used. Remarkable improvement of contrast, over $10^{-6}$, was confirmed at the area just out of IWA, which is an important area for the exo-planet search.

\subsection{CRyogenic MIR testbed}

Many of our laboratory experiments were performed at room temperature, in atmosphere, at visible wavelengths, using a monochromatic laser as a light source. Contrarily, 

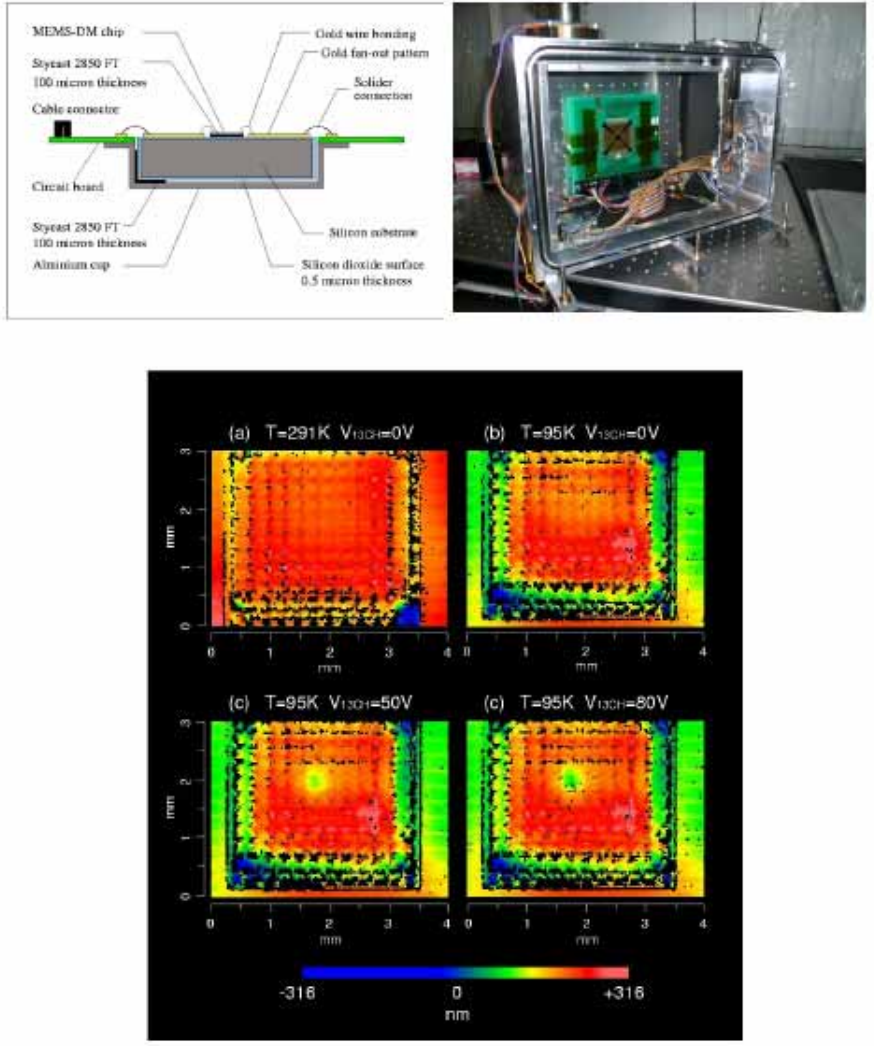

Figure 5. Top: a proto-type of MEMS cryogenic deformable mirror. Bottom: result of the demonstration of cryogenic deformation of the proto-type deformable mirror at $95 \mathrm{~K}$.

the SPICA coronagraph will finally have to be evaluated at cryogenic temperature, in vacuum, at infrared wavelengths, using a source with some band-width. We are developing a cryogenic vacuum chamber as a testbed of the whole MIR coronagraph including cryogenic active optics (Figure 7).

\section{REFERENCES}

Abe, L., Vannier, R., Petrov, R., Enya, K., \& Kataza, H. 2009, Proc. of this workshop

Burrows, A., Sudarsky, D., \& Hubeny, I. 2004, ApJ, 609, 407

K. Enya, \& SPICA working group 2009, AdSR, in press

Fukagawa, M., Itho, Y., \& Enya, K. 2009, Proc. of this workshop

Kalas, P., Graham, J. R., Chiang, E., Fitzgerald, M. P., Clampin, M., Kite, E. S., Stapelfeldt, K., Marois, C., \& Krist, J. 2008, Science, 322, 1345

Kasdin, N. J., Vanderbei, R. J., Littman, M. G. \& Spergel,D. N. 2005, Applied Optics, 44, 1117

Marois, C., Macintosh, B., Barman, T., Zuckerman, B., Song, I., Patience, J., Lafrenière, D., \& Doyon, R., Direct Imaging of Multiple Planets Orbiting the Star HR 8799, 2008, Science, 322, 1348
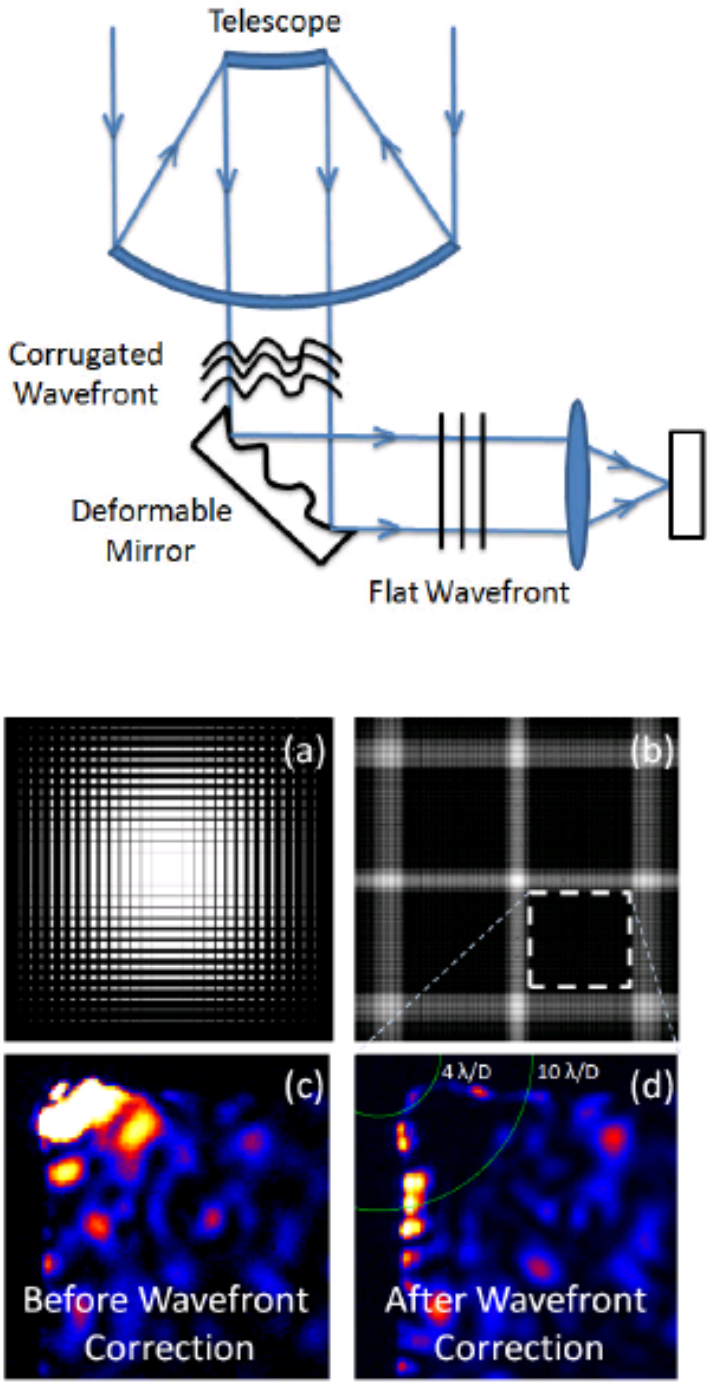

Figure 6. Study of wave-front correction algorithm ongoing with a checkerboard pupil mask and visible laser. Top: A schematic view of wave-front correction. Bottom: Panel (a) and (b) show a design of a checkerboard mask and expected PSF used for this experiment. Panel (c) and (d) show the PSF of the laboratory experiment before and after wave-front correction. Contrast higher than $10^{-6}$ (i.e., the requirement of SCI) was achieved just in outer area from IWA of the PSF.

Mayor, M., \& Queloz, D. 1995, Nature, 378, 355,

Nakagawa, T. 2009, Proc. of this workshop

Swain, M. R., Vasisht, G., \& Tinetti, G. 2008, Nature, 452,329

Tinetti, G., Vidal-Madjar, A., Liang, M.-C., Beaulieu, J.P., Yung, Y., Carey, S., Barber, R. J., Tennyson, J., Ribas, I., Allard, N., Ballester, G. E., Sing, D. K., \& Selsis, F. 2007, Natur, 448, 169

Vanderbei, R. J., Kasdin, N. J. \& Spergel, D. N. 2004, ApJ, 615, 555 


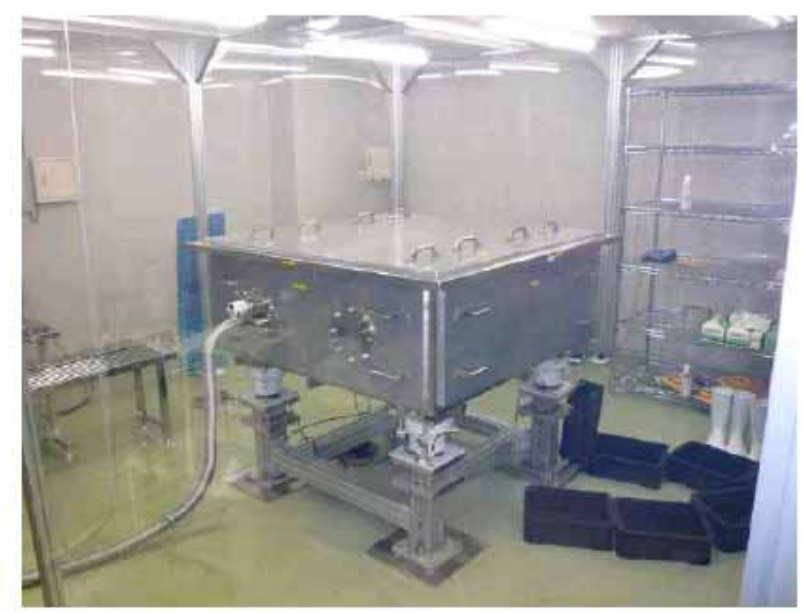

Figure 7. A cryogenic vacuum chamber as a testbed of MIR coronagraph on development.

Vanderbei, R. J. 1999, Optimization methods and software, 11, 451

Yamashita, T., Narita, N., \& Enya, K. 2009, Proc. of this workshop 